\section{P24 VITAMIN D LEVELS ARE LOW IN SARCOIDOSIS AND CONTRIBUTE TO ABNORMAL MONOCYTE ACTIVITY}

${ }^{1} \mathrm{AP}$ Crawshaw, ${ }^{2}$ YR Kendrik, ${ }^{3} \mathrm{~F}$ Martinez Estrada, ${ }^{2} \mathrm{~L}-\mathrm{P}$ Ho. ${ }^{1} \mathrm{MRC}$ Human Immunology Unit, Weatherall Institute of Molecular Medicine, University of Oxford, Oxford, UK; ${ }^{2}$ MRC Human Immunology Unit, Weatherall Institute of Molecular Medicine, University of Oxford and Oxford Sarcoidosis Service, Churchill Hospital, Oxford University Hospitals NHS Trust, Oxford, UK; ${ }^{3}$ Kennedy Institute of Rheumatology, University of Oxford, Oxford, UK

\subsection{6/thoraxjnl-2014-206260.174}

Introduction Sarcoidosis is a multisystem inflammatory disorder. We showed recently that monocytes from patients with sarcoidosis exhibited reduced IL-10 production, and were less able to suppress $\mathrm{T}$ cell proliferation ${ }^{1}$. Vitamin $\mathrm{D}$ is reduced in a number of inflammatory and autoimmune disorders and has been shown to influence the activity of immune cells, including monocytes. We had observed reduced Vitamin D levels in our sarcoidosis patients and hypothesised that this may contribute to immunepathology by altering monocyte function.

Methods Forty-six steroid-naïve, non-smoking individuals with histology-confirmed sarcoidosis were recruited from our Sarcoidosis-ILD service at first presentation. Serum calcidiol levels were compared with age and gender matched healthy controls. All donors were sampled between July and mid-October when Vitamin D levels were expected to be maximal. Monocytes were isolated using magnetic bead separation, stimulated with lipopolysaccharide (LPS) and cultured overnight with clinically 'sufficient' $\left(10^{-7} \mathrm{M}\right)$ or 'deficient' $\left(10^{-8} \mathrm{M}\right)$ levels of calcitriol. Monocyte function was assessed through cytokine production (by flow cytometry and ELISA) and multinucleate giant cell formation (MGC) (in vitro fusion assay).

Results Vitamin D (calcidiol) levels were reduced in sarcoidosis (median $25.35 \mathrm{ng} / \mathrm{ml}$ [18.43-45.05]) compared with healthy controls (median $75.3 \mathrm{ng} / \mathrm{ml}$ [IQR 52.0-88.1]; p < 0.01). For the in vitro monocyte studies, calcitriol treatment resulted in adose-dependent (i) increase in monocyte IL-10 production ([IL10 -producing monocytes, as \%total monocytes] from a baseline of10.2\% [9.4-12.0] to 27.6\% [25.6-29.4] when cultured with 'deficient' dose of vitamin D and to $30.8 \%$ [28.9-32.6]); p.

Conclusions Vitamin D levels are decreased in sarcoidosis patients at presentation. Monocyte IL-10 production was increased, and pro-TH1 differentiation cytokines were reduced with Vitamin D. Correcting Vitamin D deficiency in sarcoidosis patients may redress abnormal monocyte activity and reduce disease activity.

\section{REFERENCE}

1 Crawshaw A et al. Eur J Immunol. 2014

\section{\begin{tabular}{|l|l}
\hline P25 DISTINCT PRO-INFLAMMATORY GENE EXPRESSION \\
\hline
\end{tabular} PROFILE IN MONOCYTES FROM SARCOIDOSIS PATIENTS WITH ACTIVE DISEASE}

${ }^{1}$ AP Crawshaw, ${ }^{1}$ YR Kendrik, ${ }^{2} E$ Repapi, ${ }^{2} S$ Taylor, ${ }^{1}$ L-P Ho. ${ }^{1} M R C$ Human Immunology Unit, Weatherall Institute of Molecular Medicine, University of Oxford and Oxford Sarcoidosis Service, ChurchillHospital, Oxford University Hospitals NHS Trust, Oxford, UK; ${ }^{2}$ Computational Biology Unit, Weatherall Institute of Molecular Medicine, University of Oxford, Oxford, UK

\subsection{6/thoraxjnl-2014-206260.175}

Introduction Monocytes are potential key cellular players inthe early pathogenesis of sarcoidosis. Having previously identified altered monocyte activity in sarcoidosis, ${ }^{1}$ we now examine monocyte whole genome expression profile in order to determine potential processes and pathways involved in the perturbed immune activity of these cells.

Methods Patients with tissue-confirmed sarcoidosis (three highactivity, three low-activity, identified by a predefined clinical activity score $)^{2}$ were recruited: gene expression profiles compared with age and gender matched healthy controls. All donors were Caucasian, corticosteroid (and other immunemodulatory treatment) naïvenon-smokers. Monocytes were isolated by CD14-negative magnetic selection within $3 \mathrm{~h}$ of venesection, RNA extracted using a proprietary kit and stored at $-80^{\circ} \mathrm{C}$ prior to single batch hybridisation with Illumina HumanHT-12 v4 chips.

Results A total of 3437 genes were differentially expressed in sarcoidosis monocytes compared with controls (adjusted $\mathrm{p}$ value of $<0.05$ ). Filtering by $\log _{2}$ fold change of at least 1.5 identified 151 differentially expressed genes among these. Principal component analysis demonstrated clear segregation between sarcoidosis and controls, and between those with high and low activity, with low activity clustering closer to healthy controls. IL-6 was the most significantly upregulated gene $\left(\log _{2}\right.$ FC 4.723 adjusted $\mathrm{p}<0.001)$. Other significantly upregulated genes included the pro-inflammatory cytokines IL1A $(2.987,0.001)$ and IL1B $(2.952,0.001)$; and the monocyte chemotactic factors CCL20 (4.212, 0.002), CXCL2 $(4.057,<0.001)$ and CCL3 $(3.470,0.003)$. Gene set enrichment analysis identified gene ontology (GO) gene sets relating to inflammatory andimmune system responses to be amongst the most positively enriched genes in the monocytes from patients with active disease $(\mathrm{p}<0.001$, normalised enrichment score [NES] 2.22 and 2.20 respectively).

Conclusions Sarcoidosis monocytes sarcoidosis have a distinct gene expression profile exhibiting a pro-inflammatory, chemotactic phenotype. IL6 may be implicated in the initiation and maintenance of alveolitis and hypergammaglobulinemia in sarcoidosis and the recent interest in the use of humanised anti-IL-6R antibodies in the treatment of rheumatoid arthritis and other immune mediated disease makes functional characterisation of the role of IL- 6 in the pathogenesis of sarcoidosis an exciting prospect.

\section{REFERENCES}

1 Crawshaw A et al. Eur J Immunol 2014

2 Kendrick YR et al. Am J Respir Crit Care Med 2013;187:A1391

\section{P26 P38 MAPK INHIBITION ENHANCES CORTICOSTEROID EFFECTS IN HUMAN EPITHELIAL CELLS VIA INCREASED GR NUCLEAR LOCALISATION}

S Lea, K Gaffey, J Plumb, R Gaskell, D Singh. University of Manchester, Manchester, UK

\subsection{6/thoraxjnl-2014-206260.176}

Background Phospho-p38 MAPK expression is increased in airway epithelial cells in patients with severe asthma and in COPD patients compared to controls. Increased p38 MAPK activation may lead to reduced corticosteroid responsiveness. We have used a human cell bronchial epithelial cell line to investigate the effects of a p38 MAPK inhibitor in combination with a corticosteroid on inflammatory cytokine stimulation.

Methods The human epithelial cell line $16 \mathrm{HBE} 14 \mathrm{o}^{-}$was used to determine the effects of dexamethasone (0.1-1000 nM) alone, the p38 MAPK inhibitor BIRB-796 (1-1000 nM) alone and both drugs combined at all concentrations on LPS $(1 \mu \mathrm{g} / \mathrm{ml})$, Poly I:C 